\title{
IMAGE RESTORATION BY 1-D KALMAN FILTERING ON ORIENTED IMAGE DECOMPOSITIONS
}

\author{
M. Mattavelli M. Thonet $^{1} \quad$ V. Vaerman $^{1} \quad$ B. Macq $^{2}$ \\ ${ }^{1}$ Signal Processing Laboratory, Ecole Polytechnique Fédérale de Lausanne, CH-1015 Lausanne, Switzerland \\ ${ }^{2}$ Telecom. Laboratory, Université Catholique de Louvain, Place du Levant 3, B-1348 Louvain-la-Neuve, Belgium \\ e-mail : marco.mattavelli@lts.de.epfl.ch
}

\begin{abstract}
This paper introduces a new image restoration method based on a 1-D Kalman filtering. Using the model of tuned channels, the corrupted image is decomposed into a set of perceptual components characterized by different orientations and frequencies. The restoration step is then performed on each component in one dimension following the appropriate orientation with the well-known Kalman algorithm. Since the decomposition provides perfect reconstruction, the restored image is the recomposition of all the restored components. This approach yields relevant results for $2-D$ blurred images, using 1 -D low order models. Unlike traditional 2-D Kalman restoration techniques, its implementation has no excessive computational load.
\end{abstract}

\section{INTRODUCTION}

Kalman filtering has been used in various formulations in the field of image restoration $[1,2,3,4,5]$. The fundamental principle of this well established algorithm consists in determining recursively the causal least mean square error estimate with a two-step procedure [6]. First, a prediction of the state variables is formed on the basis of the previous state of the system. Then, the prediction is updated on the basis of the observed image data to form the estimate of the present state of the system. The update operation is achieved by means of a Kalman gain, which allows a linear correction of the previous state. This gain is computed at each iteration by a least mean square error minimization.

First applications to the restoration of blurred and noisy images were restricted to 1-D Kalman filter using 1-D image model and degradation model. [7]. Tab. 1 gives some results obtained with images corrupted by the nonseparable 2-D blurring:

$\begin{array}{lllll}0.0030 & 0.0133 & 0.0219 & 0.0133 & 0.0030 \\ 0.0133 & 0.0596 & 0.0983 & 0.0596 & 0.0133 \\ 0.0219 & 0.0983 & 0.1621 & 0.0983 & 0.0219 \\ 0.0133 & 0.0596 & 0.0983 & 0.0596 & 0.0133 \\ 0.0030 & 0.0133 & 0.0219 & 0.0133 & 0.0030\end{array}$

and restored with 1-D observation model and 1-D image model. In this case, the image is first transformed into a vector (thus neglecting the borders effect and correlation between lines) and then Kalman algorithm is applied to this vector. It clearly appears that a $1-\mathrm{D}$ restoration is not convenient when a $2-D$ degradation is present.

Following this, the approach was extended to two dimensions using a 2-D autoregressive image model with non-symmetric half plane causal supports $[1,2,4]$. This natural extension of the one-dimensional approach yields adequate results but the main problem is the excessive computational load due to the update of the state vectors depending on the size of the considered causal support. In order to decrease the complexity, reduced update Kalman filters have been proposed [1, 2, 3]. The update procedure is limited to state variables in a neighborhood of the currently processed pixel. These approaches are based on the hypothesis that a pixel has a relevant correlation only for certain neighboring pixels.

\section{RESTORATION SCHEME}

In this paper we propose a new scheme for Kalman image restoration. The corrupted image is first decomposed into tuned channels in orientation and frequency [8]. This decomposition gives rise to a set of subimages (termed the perceptual components), each of them being a representation of the degraded image around a given orientation and within a particular frequency band. The basic assumption for the definition of the causal support, image and observation models, is that in each tuned channel the correlation among pixels is relevant only in the considered orientation while it is negligible in all the other orientations. Therefore it is possible to define a causal 1-D support for each tuned channel. Obviously, the defined causal support and the scanning order depend on the orientation of the channel. This solution allows to apply simple and straightforward 1-D Kalman restoration based on observation and image models developed for each channel. Moreover the proposed choice of the causal support simplifies the boundary conditions problem that appears in reduced update 2-D Kalman approaches. Then the image reconstruction is performed by recombining all the restored perceptual components.

\section{FROM 2-D TO 1-D KALMAN FILTERING}

In order to implement the proposed scheme, a transform for the decomposition into tuned channels has been designed. This transform is defined according to the simpli- 


\begin{tabular}{||c|c|c|c|c||}
\hline \hline Image & Models orders & $\begin{array}{c}\text { PSNR blurred image } \\
(\mathrm{dB})\end{array}$ & $\begin{array}{c}\text { PSNR restored image } \\
(\mathrm{dB})\end{array}$ & $\begin{array}{c}\text { PSNR gain } \\
(\mathrm{dB})\end{array}$ \\
\hline \hline Lena & 5 & 31.19 & 29.94 & -1.25 \\
\hline Cameraman & 5 & 23.54 & 21.79 & -1.75 \\
\hline MIT & 5 & 24.32 & 21.96 & -2.36 \\
\hline \hline
\end{tabular}

Table 1. PSNR gains for 1-D Kalman restoration applied to some 2-D blurred images. The causal support follows the simple lexicographic ordering.

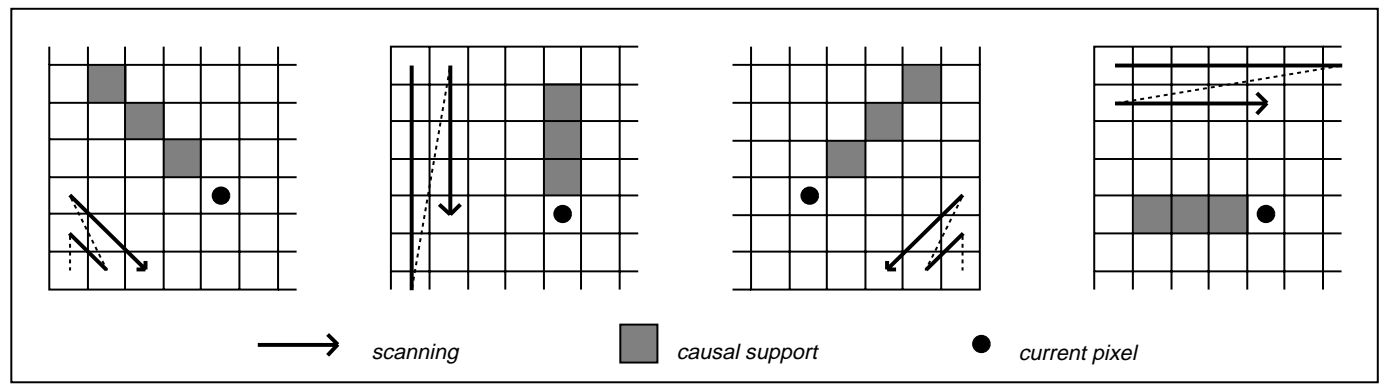

Figure 1. Causal support and pixel scanning order for the orientation at $-45,90,45$ and 0 degrees.

fication proposed in [9] for the visual perception modeling: the signal is decomposed into 4 orientations $(\theta=$ $-45,90,45,0$ degrees) and into 5 frequency bands denoted as $f=1,2,3,4,5$, plus a single DC component denoted as $f=0$. The decomposition into perceptual components is performed by filters which are separable in the polar coordinates in the Fourier domain. The impulse response of these filters is well spatially localized for the high-frequencies and well frequentially localized for the low-frequencies (octave analysis). Furthermore, the filters have symmetry and mirror properties around their cutoff frequency or cut-off angle so as to obtain the perfect reconstruction property.

The decomposition described above allows to reduce 2D Kalman restoration to 1-D in each channel. Kalman filtering requires two distinct models: an observation model which links the original image to the corrupted one, and an image model which gives the relation between the currently processed pixel and those already restored. The latter is defined as an ARMA model, with an additional term taking into account the influence of the neighboring pixels in channels with the same orientation but lower frequencies.

The first equation describes the link between the blurred component $y_{f, \theta}$ and the original component $x_{f, \theta}$ by means of the transfer function $h_{f, \theta}$. The frequency band index is given by $f$, while $\theta$ is the orientation index, and $K$ the order of the blurring. The term $w_{f, \theta}$ models the observation error as a white Gaussian noise. In the second equation, the current pixel of the original component $x_{f, \theta}$ is a combination of pixels in the causal support of the same perceptual component (autoregressive part with transfer function $a_{f, \theta}$ ) and neighboring pixels of all the components of lower frequencies $x_{\phi, \theta}$ (moving average part with transfer function $\left.c_{\phi, \theta}\right)$. Here, $v_{f, \theta}$ represents the modeling error, expressed by a Gaussian white noise. The degradation model (1) and image model (2) for each channel can be expressed by the following equations:

$$
\begin{gathered}
y_{f, \theta}(n)=\sum_{k=0}^{K-1} h_{f, \theta}(k) \cdot x_{f, \theta}(n-k)+w_{f, \theta}(n) \\
x_{f, \theta}(n)=\sum_{i=1}^{I} a_{f, \theta}(i) \cdot x_{f, \theta}(n-i)+ \\
\sum_{\phi=0}^{f-1} \sum_{j=0}^{J-1} c_{\phi, \theta}(j) \cdot x_{\phi, \theta}(n-j)+v_{f, \theta}(n)
\end{gathered}
$$

The representation of the causal support and of the scanning order for each oriented component is reported in Fig. 1. Each component is transformed into a onedimensional vector and restoration is carried out for each orientation and each frequency band, starting from the low frequencies and progressing with increasing frequency. The restored image is then obtained by recombination of all the restored components.

\section{EXPERIMENTAL RESULTS}

Experimental results have shown that the orders of the models may be kept low without loss of efficiency and that boundary effects are negligible. Tab. 2 points out the evolution of the PSNR gain with respect to the orders of the observation and image models. It clearly appears that one can restrict the orders of the models to a reasonably low value (e.g. $\mathrm{K}=\mathrm{I}=\mathrm{J}=5$ ).

The hypothesis of lack of correlation among pixels of different orientations channels has been verified. Experimental results show that the correlation with pixels of 


\begin{tabular}{||c|c|c|c|c||}
\hline \hline Image & $\begin{array}{c}\text { Observation model } \\
\text { order K }\end{array}$ & $\begin{array}{c}\text { Image model } \\
\text { order I }\end{array}$ & $\begin{array}{c}\text { Image model } \\
\text { order J }\end{array}$ & $\begin{array}{c}\text { PSNR gain } \\
(\mathrm{dB})\end{array}$ \\
\hline \hline Lena & 3 & 3 & 3 & 3.05 \\
\hline Lena & 5 & 3 & 3 & 3.08 \\
\hline Lena & 5 & 5 & 5 & 3.14 \\
\hline Lena & 7 & 5 & 5 & 3.16 \\
\hline Lena & 9 & 5 & 5 & 3.17 \\
\hline \hline
\end{tabular}

Table 2. PSNR gains for some orders of the image and observation models.
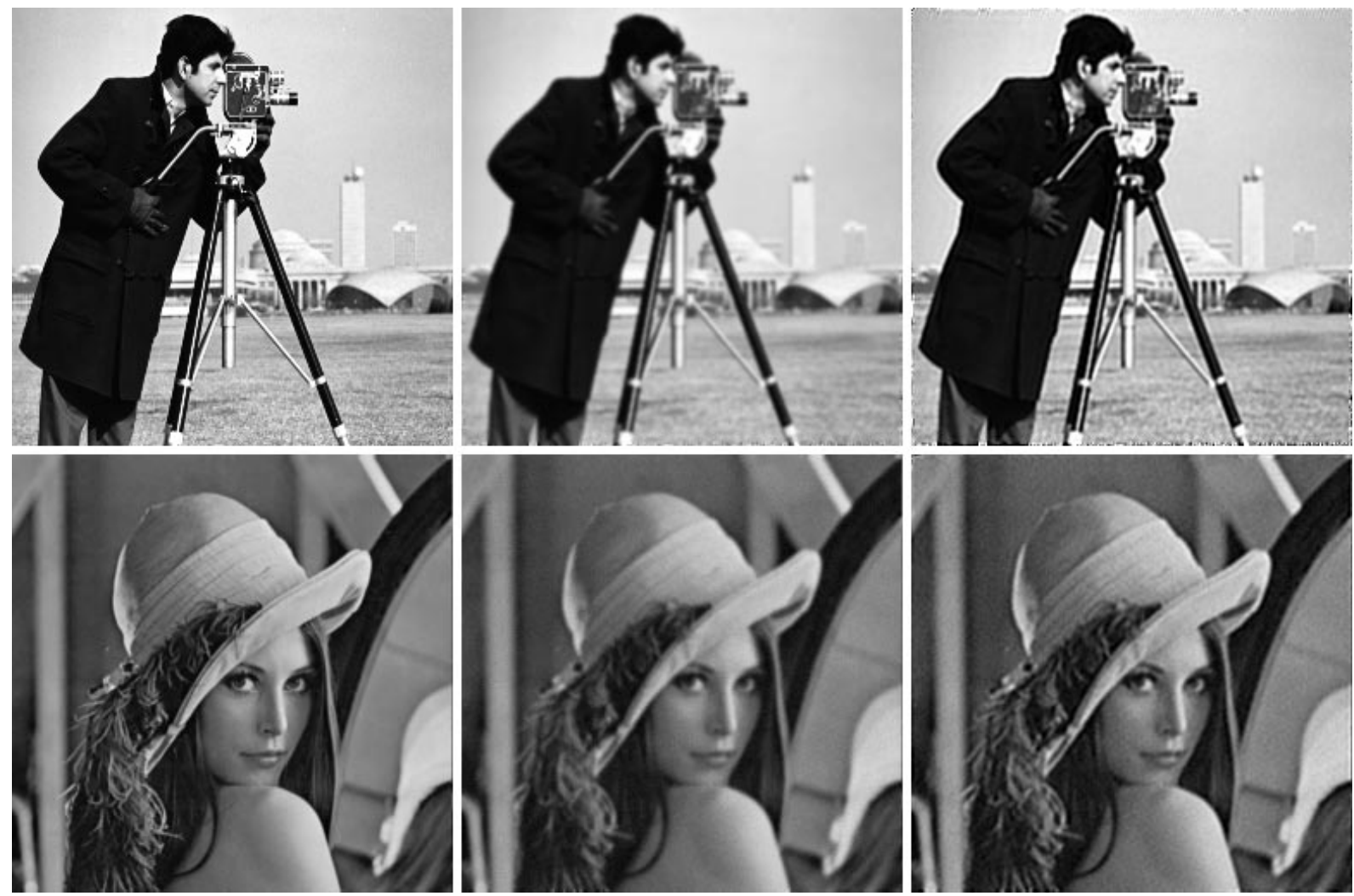

Figure 2. Original image (left), blurred image (center) and restored image (right) by 1-D Kalman filtering on oriented components. The images are degraded by a 2-D blurring, and a white noise of variance 9 has also been added to the image Lena.

\begin{tabular}{||c|c|c|c|c||}
\hline \hline Image & Noise var. & $\begin{array}{c}\text { PSNR blurred image } \\
(\mathrm{dB})\end{array}$ & $\begin{array}{c}\text { PSNR restored image } \\
(\mathrm{dB})\end{array}$ & $\begin{array}{c}\text { PSNR gain } \\
(\mathrm{dB})\end{array}$ \\
\hline \hline Lena & 0 & 31.19 & 34.33 & 3.14 \\
\hline Cameraman & 0 & 23.54 & 25.93 & 2.39 \\
\hline Lena & 9 & 30.48 & 32.50 & 2.02 \\
\hline Lena & 100 & 26.41 & 29.09 & 2.68 \\
\hline \hline
\end{tabular}

Table 3. PSNR results and improvements for two images, with and without additive white noise. 
other orientation channels yields coefficients whose values is indeed negligible.

Different kinds of blurring have been applied to some test images. White Gaussian noise has been also added to the corrupted images to confirm the robustness of the new method. Relevant reconstruction PSNR gains are obtained in the cases with and without the addition of Gaussian white noise. Some typical results are reported in Tab. 3.

Fig. 2 reports an example of the restoration for Cameraman and Lena images.

\section{CONCLUSION}

In this paper, a new method for 1-D image restoration by Kalman filtering has been developed. The 1-D filtering operation is carried out by decomposition of the blurred image into subimages by means of the tuned channels model. According to this model, each of these perceptual components represents the original image within a band of frequency and around a given orientation. Since information in each component is found to be relevant only in one direction, the restoration process can be achieved by a straightforward 1-D Kalman algorithm. The restored image is then reconstructed by a simple addition of all the restored components. The main advantage of this method, in comparison with classical 2-D techniques, is to avoid a heavy computational load occuring when 2 -D restoration techniques are implemented.

Experimental results have shown that significant PSNR gains are obtained, even with observation and image models of low order (typically 3 or 5 ). Moreover, robustness has been underlined by the addition of white noise on a broad variance scale.

Further improvements of the method can be considered, especially for the determination of optimal orders and supports, as well as the characterization of the mutual influences of the perceptual components concerning the image model. A future extension is also considered in order to apply the method to other kinds of degradations and not only non-separable blurring.

\section{REFERENCES}

[1] J.W. Woods and C.H. Radewan. Kalman filtering in two dimensions. IEEE Transactions on Information Theory, IT-23(4):473-482, July 1977.

[2] J.W. Woods and V.K. Ingle. Kalman filtering in two dimensions: Further results. IEEE Transactions on Acoustic, Speech and Signal Processing, ASSP29(2):188-197, April 1981 .

[3] J. Biemond, J. Rieske, and J.J. Gerbrands. A fast kalman filter for images degraded by both blur and noise. IEEE Transactions on Acoustic, Speech and Signal Processing, ASSP-31(5):1248-1256, October 1983.

[4] A.M. Tekalp, H. Kaufman, and J.W. Woods. Identification of image and blur parameters for the restoration of noncausal blurs. IEEE Transactions on Acoustic, Speech and Signal Processing, ASSP-34(4):963971, August 1986.
[5] M.I. Sezan and A.M. Tekalp. Survey of recent developments in digital image restoration. Optical Engineering, 29(5):393-404, May 1990.

[6] R.E. Kalman. A new approach to linear filtering and prediction problems. Journal of Basic Engineering, 82D:34-45, 1960 .

[7] S.S. Dikshit. A recursive window approach to image restoration. IEEE Transactions on Acoustic, Speech and Signal Processing, ASSP-30(2):125-140, April 1982 .

[8] B. Macq, M. Mattavelli, O. van Calster, E. van der Plancke, S. Comes, and W. Li. Image visual quality restoration by cancellation of the unmasked noise. In IEEE, editor, Proceedings of ICASSP 94, volume 5, pages 53-56, Adelaide, Australia, April 1994.

[9] S. Comes and B. Macq. Human visual quality criterion. In M. Kunt, editor, Visual Communications and Image Processing '90, volume 1360, Lausanne, Switzerland, October 1-4 1990. 